\title{
Biophotons: Ultraweak Light Impulses Regulate Life Processes in Aging
}

\section{Hugo J Niggli*}

BioFoton AG, Rte. d'Essert $27 \mathrm{CH}-1733$ Treyvaux, Switzerland

\begin{abstract}
As shown by the history of light, photons participate in most atomic and molecular interactions. Recent biophysical research has measured low light impulses, so-called biophotonic emission, in cells and biological tissue. It is reported throughout the world that all cells (plant, animal or human) emit a weak, so-called biophotonic radiation. Based on the photoelectric effect, appropriate photomultiplier systems have been developed in order to detect this very weak light. Although the emission is extremely low in mammalian cells, it can be efficiently induced by light leading to delayed luminescence or light induced ultraweak photon re-emission. Re-emitted photons in cells are coupled with radical reactions and are probably also linked with the DNA as an important source. In recent years, cell culture models for biophotonic measurements using fibroblastic differentiation were generated and were used as an example to test the growth stimulation efficiency of various bone cell growth factors. It is well known that fibroblasts play an essential role in skin aging, skin carcinogenesis and wound healing. Therefore the biophotonic model of cells provides a new and powerful non-invasive tool for the development of new strategies in aging research.
\end{abstract}

Keywords: Aging; Biophotons; Photon; Photomultiplier; Photo electric effect; Fibroblast; Skin; Human; Ultraviolet; DNA changes

\section{History of Light}

The high importance of electromagnetic radiation for human life is well known. Following winter season, life on our earth retrieves vitality in early spring mostly by the sunrays reaching the ground. When white light enters a prism, it will divide into the well-known spectral colours: From red to orange, followed by yellow, changing to green as well as blue and finally showing the violet dye. The scientist Isaac Newton (1642-1727) noticed this already in his mid-twenties [1]. This observation indicated that white light was a composition of the rainbow colours. Johann Wolfgang von Goethe (1749-1832) confirmed this discovery. The light spectrum is not exclusively detected in the rainbow. In the action of forging one can notice that iron, once it is heated by the fire, switches colours from dark to bright red. It ultimately alternates at last into white. Although not combustible, solid matter behaves in this manner. From this observation, it is clear that long-wavelengths appear at lower temperatures. As the temperature elevates, shorter wave-lengths are noticed until all wave-lengths are present in the incandescent substance. Examinations on extremely hot stars have demonstrated that long wave-lengths steadily vanish and the colour changes into the blue spectrum range. The quantum theory of light is based on the scientific findings of Max Planck (1858-1947) as well as Albert Einstein (1879-1955) and clarifies the nature of light [1]. They demonstrated the particle- and wave-like qualities of light. In 1803, the scientist Thomas Young (1773-1829) showed evidence of interference patterns in visible radiation [1]. His experimental testing demonstrated that visible radiation is based on waves. This has already been shown by Christiaan Huygens (1629-1695). The electromagnetic theory of light was stated by James Clerk Maxwell (1831-1879). He explained light as a composition of electric and magnetic fields. In 1900 the physicist Max Planck postulated that the electromagnetic spectrum (Figure 1) is based on small discrete packets. These quanta are called photons, the most minuscule light particle [1].

Albert Einstein utilized this quality of light in order to explain the photoelectric effect. The Nobel Prize Committee decided in the year 1922, to honour Einstein for this discovery with the still open Nobel Prize in Physics of the year 1921 and not for his brilliant relativity theory developed a decade later [1]. As a practical application, these theoretical findings on photoelectric effects allowed to quantify photons by sensitive photomultiplier systems. By this sophisticated method it is possible now to detect a firefly at a distance of six and $4000 \mathrm{~km} \mathrm{[2].} \mathrm{Another} \mathrm{application} \mathrm{of} \mathrm{this} \mathrm{discovery} \mathrm{can} \mathrm{be} \mathrm{seen} \mathrm{in} \mathrm{the}$ automatic closure of the door of a lift. A beam of light in the door system hits the metal plate and therefore an electric current starts to flow in order to close the door. As soon as this process is interrupted by a person entering, this electric current stops and it is for this reason the door stays open [3].

\section{The Earth's Electromagnetic Spectrum and the Interaction of UV Radiation with Molecules of Biological Importance}

A great electromagnetic energy field surrounds our earth. Figure 1 depicts the broad electromagnetic spectrum arriving on the surface of the earth originating from the sun and space. Parrish et al. [4] as well as Harm [5] gave relevant background information on electromagnetic radiation with high priority of the interaction of UV-irradiation with molecules of biological importance. I summarize their mentioned general aspects on electromagnetic radiation as follows:

The electromagnetic radiation shows distinct frequencies from cosmic- to gamma rays, followed by X-rays and ultraviolet light. UV light is subdivided into three electromagnetic wave ranges: UVA (320$400 \mathrm{~nm})$, UVB $(290-320 \mathrm{~nm})$ and UVC $(<290 \mathrm{~nm})$. This terminology was originally defined by Coblentz et al. in the year 1932, on the basis of physical- and biological effects of these ultraviolet regions as summarized elsewhere. It is scientifically shown, that the ozone layer in the stratosphere absorbs almost all short wave-lengths of ultraviolet light (UVC) in order to avoid changes in DNA structures of living organisms [6]. Obviously it is necessary for life on earth that potentially harmful high energy radiation is absorbed by the atmosphere of the earth. As summarized before [6], radiation from the ultraviolet region

*Corresponding author: Hugo J Niggli, Rte. d' Essert 27, CH-1733 Treyvaux Switzerland, Tel: 41264131445; Fax: 41264131445; E-mail: biofoton@hispeed.ch

Received October 27, 2013; Accepted January 07, 2014; Published January 09 2014

Citation: Niggli HJ (2014) Biophotons: Ultraweak Light Impulses Regulate Life Processes in Aging. J Gerontol Geriat Res 3: 143. doi:10.4172/2167-7182.1000143

Copyright: (ㅇ 2014 Niggli HJ. This is an open-access article distributed under the terms of the Creative Commons Attribution License, which permits unrestricted use, distribution, and reproduction in any medium, provided the original author and source are credited. 


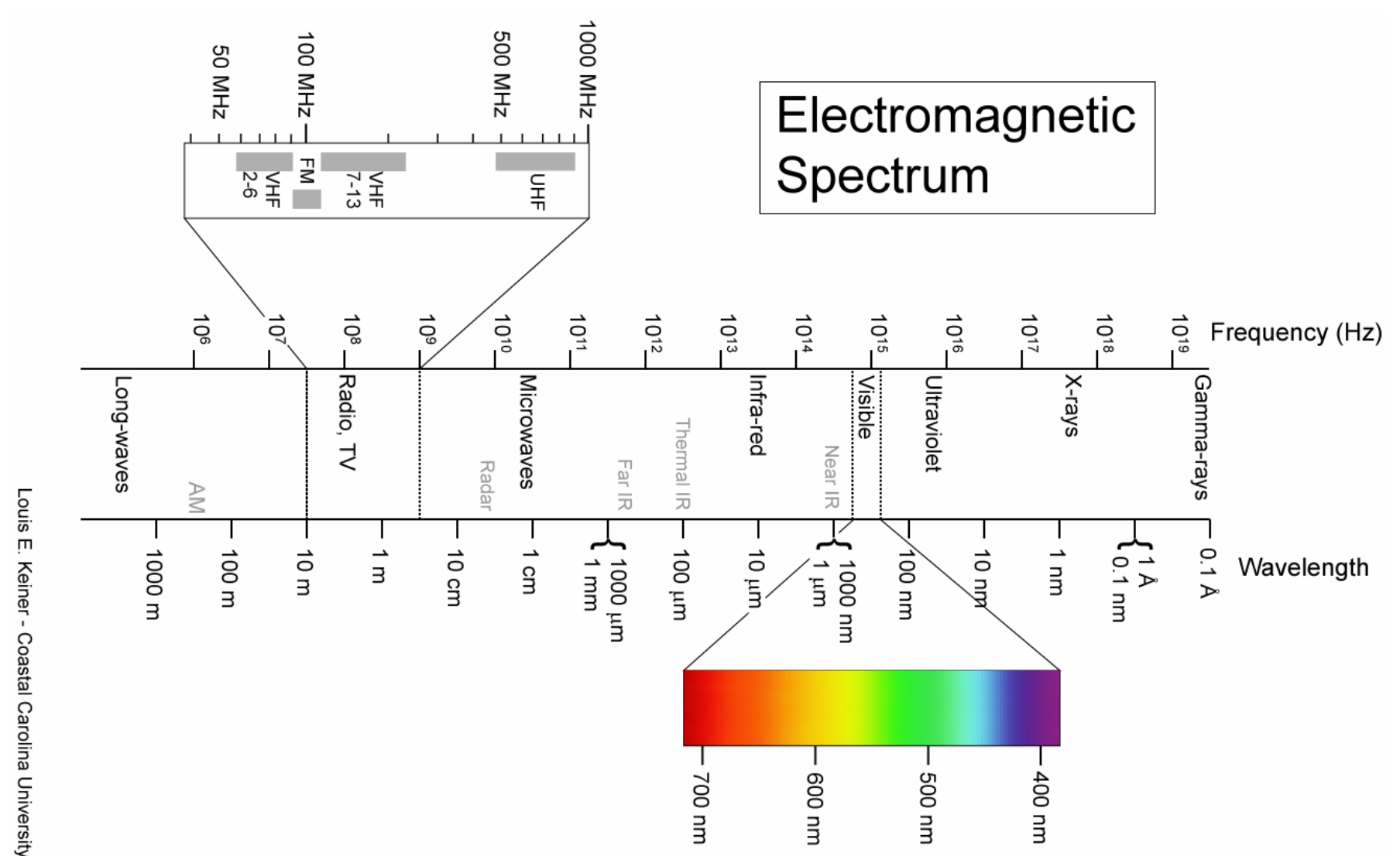

Figure 1: Electromagnetic spectrum.

down to all less energetic radiation (Figure 1) is referred to non-ionizing radiation as opposed to ionizing radiation. The latter is represented in the EM spectrum essentially by X-rays, alpha-rays, beta-rays and gamma-rays, which consist of charged particles. The reason for this distinction is the different interaction with matter: Ionizing radiations are capable of charging all kinds of atoms and molecules. The radical processes induced by ionising irradiation can change, damage and even destroy functions of the cell $[4,5]$. Just remember the destructive events in August 1945 induced by such radiation through the atomic bombs in the Japanese towns of Hiroshima and Nagasaki. Most recently in the Fukushima Daiichi nuclear disaster similar effects were provoked. On the other hand, the healing effects of radioactive thermal sources are also well known.

In contrast, absorption of nonionizing radiations typically leads to electronic excitation of atoms and molecules as described in detail by Parrish et al. [4] as well as Harm [5]. The absorption of UVC radiation by the ozone layer is in contrast to the open window (defined as solar electromagnetic radiation which reaches the earth surface at almost $100 \%)$ in the range of visible light. Other open atmospheric windows are located in the infrared region and in the electromagnetic spectral part of radio waves (Figure 1).

The most important biological effects are known up to date by the interaction of UV radiation with molecules of biological importance. Primarily these effects induced by UV-light are due to its absorption by nucleic acids and to a significantly lower extent by proteins and other biologically important molecules like for example vitamins. The main reason for this practical observation is the following: In contrast to all the nucleotide residues in DNA, only the aromatic amino acid residues of proteins absorb measurably in the UV region from 240-300 $\mathrm{nm}$. Biological substances, other than nucleic acids, like for instance flavins, porphyrines, steroids, quinones or carotenoids, may be sometimes involved in the biological effects induced by UV light. The absorbing components of UV light within nucleic acids are the bases (pyrimidines and purines). They possess an absorption maximum in the $260-265 \mathrm{~nm}$ region and their absorption declines rapidly toward the UVA-region. However, the DNA bases still can be induced by UVB and UVA light via radical induced processes, an energy transfer called photosensitization. The absorption characteristics of RNA resemble those of DNA. But it is well known that UV-induced changes in RNA are of significant less biological importance than similar alterations in DNA. The main reason is the uniqueness of the latter molecule as genetic material. All of the major photoproducts such as pyrimidine photodimers, pyrimidine adducts, pyrimidine hydrates and the socalled "spore photoproduct", all reviewed in the doctoral thesis of Niggli [6], turned out to be pyrimidine derivatives (including those whose biological relevance has been definitively demonstrated). Photodecomposition of the purines, the other part of DNA bases by UVC light, as judged by alterations of their absorption characteristics, occurs with lower quantum yields than those of pyrimidine (1-2 orders of magnitude lower). For this reason, almost no work has been performed on purine photoproducts, even though their possible contribution on biological effects cannot be ruled out [5].

As summarized by Niggli [6] the most significant breakthrough in DNA photochemistry was the isolation and identification of the thymine dimer from UV-irradiated frozen thymine solution. This observation was made simultaneously in the year 1960 by Beukers 
and Berends as well as Wang. The first chemical evidence for the structure of thymine photodimers derived from DNA was reported in the year 1966 by the scientists Blackburn and Davies. Cyclobutanetype photodimerization in DNA was shown in the same year by the researchers Setlow and Carrier to extend to pyrimidines in general. It is also shown that in double stranded UV-irradiated DNA mostly the cis-syn stereoisomer of pyrimidines is formed [6].

Research of electromagnetic wavelengths in the lower energy spectral range is minimal. Nevertheless, this radiation points to interactions of biological importance. Rodemann et al. [7] and scientists in the field of cell biology, reported accelerated aging and differentiation of skin cells by low energy electromagnetic fields [7]. But also the influence of mobile phones on the human health has to be considered as reported by Hyland [8] a pupil of Herbert Fröhlich. He discussed in his report published in 2000 that low energy irradiations by mobile phones may induce biological effects [8].

\section{A Historical Survey of the Quantum Field}

The French physicist and engineer Augustin Jean Fresnel (17881827), did basic work on the wave theory of light. According to Aristotle's fifth element aether, he termed it also aether [1]. The Greek philosopher Aristotle (384-322 B.C.E.) classified creation into an earthly-and a heavenly-world. He stated that the heavenly, invisible world is the region of aether. In contrast, the visible world is the one of the four elements earth, water, air and fire. In the cosmogenic theory, originated by the Greek pre-Plato philosopher Empedocles (ca. 490430 B.C.), these four elements were already defined as highly important. Aristotle clearly differentiated aether from the condensed matter of the apparent world and also from the hidden world. His idea of aether is obviously based on subtle energies [1].

The American physicist Albert Abraham Michelson (1852-1931) received the Nobel Prize in Physics in 1907. In collaboration with Edward Morley (1838-1923), they tried to demonstrate in 1887 that light needs aether as a medium [1]. In order to prove this hypothesis, they measured the speed of light in the two vertical directions using an interferometer. Surprisingly, they found no difference within the experimental error detected in both cases. In 1905 Albert Einstein tried to solve the problem as depicted briefly (1): He described light behaviour as both particle and wave. As a consequence, a particle does not need a medium as a wave normally requires. Nevertheless, in the year 1920, Albert Einstein himself proposed a kind of immeasurable aether in the form of very fine matter [9]. He then stated the unity of space, objects and events. This approach opened the door for an intensive examination of the qualities and structural properties of empty space itself. Lambrecht reported this quantum vacuum state in 2002 [10]. This new aether concept links back to the ancient Greek idea of Aristotle. This new subtle energy appears in modern science as the zero-point energy (ZPE) of the vacuum $[2,11]$. The bases of the quantum field began, like the origin of the quantum theory itself, with the brilliant idea of Max Planck that the emission of energy by a resonator can only occur in discrete packets [1]. Therefore, he proposed in 1912 the existence of a zero point field [2,11]. Walter Nernst (1864-1941), the physical chemist and Nobel Prize Laureate in chemistry in 1921, continued that of Planck's lead. He also claimed that in empty space no matter or radiation can be observed. Nevertheless, at the absolute zero point $\left(-273.15^{\circ} \mathrm{C}\right)$, energy still persists. Based on the quantum theory, at this absolute zero point, there is however motion in an atom, a molecule or a crystal. As a consequence of this consideration, the empty space in a physical system shows constant activity and owns ZPE $[2,11]$. The meaning of this energy can be best visualized as oversized model of the simple hydrogen atom: In the middle of a town we see the church as symbol for a proton. A grain of sand one and a quarter miles apart from this centre represents an electron. The empty space around the proton is a field of zero point energy. As reviewed by Mc Taggart in 2003 [11], the physicist John Archibald Wheeler (1911-2008), the Wolf Prize winner in Physics of 1997, stated that for the physics of the universe there is a need of information. He estimated $10^{115}$ Joules for the zero point energy in empty space per one cubic centimetre. This is more energy than the whole energy of all the condensed matter of our known universe. Even though further estimations resulted in significantly lower values for the energy concentration of this zero point vacuum field, the imagination of this immense magnitude shows that a tumbler of empty space contains enough energy to bring an ocean like the Pacific to a boil. Hal Puthoff, born in 1936, reported in the late that matter needs a dynamic interaction between the ZPE and subatomic particles in order to subsist in a stable situation [11,12]. Through mathematical computations he also supposed that the fluctuations of the waves of ZPE in the vacuum drive the movements of subatomic particles. Furthermore he proposed that all the movements of every elementary particle in our universe create the ZPE. In his view, the ZPE comprises the entire cosmos. Following to this speculation everything in the universe is linked with the far corners of the cosmos by the waves of the ZPE $[11,12]$.

\section{Cell cultures as Important Tools for Cell Research on Aging}

Living organisms as prokaryotes which include bacteria and archaea as well as plants, animals and humans, classified as eukaryotes, consist of cells. In protozoa, the entire organism consists of a single cell, whereas in multi-cellular metazoans, cells are the fundamental structural unit of which the entire plant, animal or human body is build up. This cellular unit depends on function and location within the cellular organism. Cell size is generally between 10 and $100 \mu \mathrm{m}$. Eggs are the largest cells formed in animals. The number of cells in an organism is closely related to the body size. An adult human individual will consist of around 100 trillion $\left(100 \times 10^{12}\right)$ cells. Cells can be grown as tissue cultures outside the organism, a technique developed by Alexis Carrel (1873-1944), the Nobel Prize winner for medicine and physiology in 1912 [1]. Although this famous French scientist claimed that cell cultures are immortal, Leonard Hayflick and his co-worker Paul Moorhead have reported in the 1960's that fibroblasts are aging and have a limited number of cell divisions before death as summarized before $[6,7,13]$. Last but not least, cell cultures have gained in importance recently for biological, biochemical and medical investigations of animals and humans.

This technique was used for example to investigate the biological effect of ultraviolet light (UV) on prokaryotic and eukaryotic cells. UVC and UVB are able to induce mutations of the genome. Therefore, it can easily cause skin cancer after intense exposure to the sun in individuals suffering from Xeroderma Pigmentosum. The biochemical defect of DNA excision repair was found to be the cause of this well investigated genetic disease in 1968 by James Cleaver in San Francisco, USA [14]. Interestingly, such genetic mutations are reversible by UVA and violet light. The so-called photo repair is a very important DNA recovery system intensively studied for example by Richard Setlow, the scientific pioneer discovering pyrimidine photodimers in DNA after ultraviolet exposure as reviewed elsewhere [6].

Cell culture technique allows human or animal cells to stay alive outside of the organism. They can proliferate during many generations. A prominent example of cell cultures are skin cells which are very easy to be grown in tissue culture flasks. The middle part of the skin, the 
so-called dermis contains mainly fibroblasts, which are most suitable to be cultivated. Skin fibroblasts will differentiate similar to the development of blood cells. Starting from an omnipotent stem cell, a highly differentiated, specialized cell will develop. The old fibroblasts are not able to divide any more. A highly complex fibroblastic model system for cell differentiation, aging and cancer was developed by the German cell biologist Klaus Bayreuther at the end of the 1980s [7,15]. Hayflick [13] was the pioneer for fibroblastic aging models in the early 1960s. Since this time human diploid fibroblasts have become a widely accepted in vitro model system. Bayreuther and co-workers extended this experimental approach showing that fibroblasts in culture resemble, in their design, the hemopoietic stem-cell differentiation system $[7,15]$. In their reports morphological and biochemical evidence for the fibroblast stem-cell differentiation system in vitro was elaborated. They showed that normal human skin fibroblasts in culture spontaneously differentiate from mitotic fibroblasts (MF) to post-mitotic fibroblastic stages (PMF). In their work methods have been developed to shorten the transition period and to increase the frequency of distinct postmitotic cell types using physical methods like ultraviolet light or chemical agents such as mytomycin C, 5-fluorouracil, and 5-bromo-2deoxyuridine $[7,15,16]$. Mitomycin $C$ is an effective chemotherapeutic agent for several cancers in man. Most proably this effect is based on the interaction with DNA. This will lead to DNA-DNA- as well as DNA-protein-crosslinks. We have demonstrated in the early 1990s that the mitomycin C-treatment of three different normal human fibroblast strains (CRL 1221, GM 38 and GM 1717), frequently used in aging research, induce characteristic morphological changes in the fibroblasts [15]. The chemical treatment induces specific shifts in the $\left[{ }^{35} \mathrm{~S}\right]$ methionine polypeptide pattern of total cellular proteins. Using this aging model system, we were also able to demonstrate that no significant difference exists in the rate and the extent of the excisionrepair response to thymine-containing pyrimidine dimers following UV-irradiation shortly after mitomycin C treatment of distinct strains of human skin fibroblasts and in the mitomycin C-induced PMF stage of these cells $[15,17]$. In addition, aphidicolin inhibits excision repair of UV-induced pyrimidine photodimers in low serum cultures of mitotic and mitomycin C-induced postmitotic fibroblasts of human skin [18]. Since fibroblasts play an essential role in skin aging, and wound healing, our results imply that the fibroblast differentiation system is a very useful tool to unravel the complex mechanism of skin aging.

\section{The Biophotonic Light in the Cells}

In 1983, the two natural scientists Nagl (cell biologist) and Popp (biophysicist) introduced in biological research an electromagnetic model of cell differentiation [19]. This model was based on the finding, that the ultra-weak radiation of cells can be measured by the technique of sophisticated photomultipliers used normally in physics to unravel the subatomic structure. The physical basis of this highly sensitive analytical device is the photoelectric effect introduced by Albert Einstein in 1905 [1]. To detect single photons, in the early 1950s the photomultipliers tubes were developed. The mechanisms of this technique in short: An electron is emitted after the absorption of a photon by a photomultiplier cathode. This electron is amplified like an avalanche by several series-connected dynodes. Finally, the resulting electron stream hits the anode and is registered as an electric impulse $[2,20]$. With this technical device, the Italian physicists Colli et al. were already able to provide evidence of ultra-weak light in plant cells in the middle of the 1950s [21]. Even earlier, at the beginning of the1920s, the Russian scientist Alexander G. Gurwitch (1874-1954) discovered this ultra-weak cell radiation in dividing onion cells microscopically. He observed different numbers of mitotic cells in onion roots which were separated by glass (protection of UVC and UVB light), compared to those separated by quartz (transmission of UV light). Based on these differences, he postulated that living organisms communicate via UV-light exchange $[2,19,20,22]$. This concept was corroborated by the Austrian physicist Erwin Schrödinger, who obtained the Nobel Prize in physics in 1933. This physicist is actually considered as the originator of quantum theory [1]. Schrödinger proposed that in living cells the high level of organization can only be maintained because the cellular system perpetually obtains order from the environment. According to Schrödinger sunlight provides this miraculous order $[2,20]$. In the1950s, the physicist Herbert Fröhlich (1905-1991) completed this genial idea by introducing the concept of coherence into living systems as summarized by his pupil Hyland [8]. He recommended light with a high degree of organization: The so-called biological laser light. The ultra-weak radiation of such a system is very calm and shows a stable intensity. The fluctuations normally seen with light are minimal. Based on the stable field strength of its waves, they are able to superpose. The light field of such a laser exhibits a high degree of order and therefore is able to generate coherence and to transfer information. In the early 1970s, the German biophysicist Fritz-Albert Popp, the Japanese researcher Inaba and the Australian natural scientist Quickenden independently provided evidence for these postulated light fields in plant, animal and human cells using the highly sensitive photomultiplier technique reviewed elsewhere [22]. This was the confirmation of the cell radiation proposed by Alexander Gurwitch using modern scientific equipments. Fritz-Albert Popp proposed to call this cell radiation biophotons (derived from the Greek "bios": life and "phos": light, power). This new biophotonic research showed, that all living organisms emit a weak, coherent light which generates order and information in the cells to regulate inner processes and actions [2,19-22].

Fritz-Albert Popp, the theoretical biophysicist from Marburg, was mainly interested in the interactions of light and biological systems. As summarized by Mc Taggart [11], he worked as a student in the same house, sometimes even in the same room, as Wilhelm Röntgen (1845-1923). This scientist discovered that X-rays are able to generate images of our body's skeleton. For this discovery he received the Nobel Prize in the year of 1901. At the same university, Popp has developed a method of irradiation that could predict the carcinogenic potential of chemicals: He found that carcinogens absorb ultraviolet-A light (UVA) in the range of $380 \mathrm{~nm}$ and change, at the same time, the frequency [11]. This hypothesis in the year of 1975, that ultra-weak UVA-light was produced somewhere in the body, was breaking a dogma. If light does exist in the body, why did not natural scientists unravel this enigma before?

In order to prove that cells are light emitters, Popp together with the young physicist Bernhard Ruth constructed a highly sophisticated, analytical device for the detection of ultra-weak radiation. The young German scientist Ruth [23] carried out the first $\mathrm{PhD}$ thesis in the field of biophotonic light under the supervision of Fritz-Albert Popp. Using a very sensitive photomultiplier tube, the two scientists were able to measure light emitted by a candle at a lunear distance of $384^{\prime} 400$ $\mathrm{km}$. The first biophotonic experiment with plant cells was performed in 1976. Ruth had grown cucumber seedlings and added them into the measurement chamber of the highly sensitive device based on photomultiplier technique. He surprisingly found that the germinated plant seeds emitted light of an astonishingly high intensity. Ruth was extremely sceptical and thought that this was emitted by chlorophyll, the basic molecule for photosynthesis which is responsible for the green stain of plants. In order to exclude this possibility, the two 
researchers decided to use potato seedlings for their next experiment. They could cultivate this plant cells in the dark. Nevertheless, their sensitive photomultiplier system registered light quanta and to their surprise the intensity was even higher than in the cucumber seedlings. Therefore, the hypothesis of the interfering photosynthetic molecule of chlorophyll could be excluded [23].

As already mentioned above, the Austrian physicist Erwin Schrödinger postulated that living cells conserve their high level of organization by sucking order from the environment [20]. According to Schrödinger it is the sunlight, which finally provides this order in biological systems. However, is it possible to measure this regulative force in living organisms? Popp and his co-workers found in their biophotonic measurements of plant cells the highest possible degree of quantum order. In other words a coherent ultra-weak radiation can be observed in living systems. Normally, such a coherent behaviour-the so called Bose-Einstein condensation - can only be found in superconductors, which are investigated in the laboratory at temperatures near the absolute zero point. As already described before, in 1950 the physicist Herbert Fröhlich found the explication of super conduction which has been discovered in 1911. By this discovery he revolutionized the field of biophysics. He introduced the concept of the coherence in living systems $[8,20]$. To get an idea of such a coherence one can imagine the following: Envisage the surface of a lake which is covered by a completely steady pattern of water waves. All these waves are of the same height, the same distance between each other, the same velocity and the same direction. It is possible to induce the circles of such waves by a stone which is thrown into the water. But as soon as on this lake suddenly a wind begins to blow, the waves will run in various directions back and forth, crossing, superposing or canceling out each other in a short time, which can be compared with normal non-coherent light. From this picture it becomes also clear, that information is transmitted best, depending on the steady state of waves. According to Herbert Fröhlich, the coherent radiation of light in biological systems is very calm, featuring a stable intensity and a minimal fluctuation. Based on the stable field strength of its waves, they are also able to superpose. The light field of such a laser exhibits a high degree of order and therefore is able to generate order and to transfer information in living systems $[2,7,20]$.

In the middle of the nineties of the last century, Fritz-Albert Popp and the chemist Sophie Cohen [24] measured for the first time light emission on man. They showed biological daily rhythms by biophotonic measurements on the hands and the front of a human individual. There was a correlation between right and left hand. Relating to light, the right hand knew what the left did. In addition, the emissions followed further biological rhythms. There were similar night, day, week and month values as if the body would follow the biorhythm of the world in connection with its own. Popp found on healthy persons a high dependency of coherence on the quantum level (ultraweak photon emission). In short, both spontaneous as well as light induced biophotonic emission were significantly correlated between left hand and right hand values. Most interestingly, in a case of multiple sclerosis this coherence was highly disturbed.

As described before, the doctoral thesis of Ruth [23] in the year of 1977 was the hour of birth of biophotonic analysis [23]. At the beginning of the eighties of the twentieth century Popp and his colleagues developed a model to demonstrate the regulation in living systems by ultra-weak radiation $[2,19,25]$. Their hypothesis is based on the assumption that in the nucleus the helically shaped genetic material acts as a biological laser obtaining its energy from the cellular nutrients in the form of photons. The Russian chemist Voeikov [26] proposed a few years ago that radical reactions from oxygen in water are inducing photons [26]. From the work of Popp and his co-workers, it became clear as well, that normal human cells have the capacity to accumulate the ultra-weak energy of light transferred to them and to utilize it for their own complex processes of life. Dying cells for instance, loose this ability of light memorization and show before death a significant increase of ultraweak photon emission. In short, their capacity to store energy of light is lost. A similar event although much slower is happening in the cells during the processes of aging. Furthermore, in the case of cancer cells, the cellular toxins accumulated during the years, and often leading to deposits of cellular debris in the tissues (e.g. arteriosclerosis in blood vessels), induce the increased emission of biophotons $[27,28]$.

Karl-Heinrich Müller who died in 2007, is the founder and initiator of the Island of Museum and Art "Hombroich", a paradisiacal garden and dreamland of wonderful beauty. Fritz-Albert Popp and Karl-Heinrich Müller established in the mid-nineties a Center of Biophotonics at a former rocket station close to this island of art (in Neuss nearby Düsseldorf, Germany). This scientific biophotonic center connects a worldwide network of scientists from Canada, China, England, Holland, Germany, Japan, India, Italy, Russia, Switzerland, Ukraine and the United States of America. In the years between 2001 and 2005, the analysis of biophotonics was brought to the highest level of photomultiplier technique with the ARETUSA method. The biophysicist Francesco Musumeci from Catania (Italy) and his co-workers at the Sicilian Center of Nuclear Science (LNS-INFN) $[28,29]$ developed a new highly sensitive analytic photomultiplier system allowing for the first time to measure the spectrum of ultraweak photon emission in individual human cells. These researchers achieved a crucial improvement of the technique of ultra-weak light measurement [29]. The main peak of the spectral distribution light was at approximately $550 \mathrm{~nm}$ [29]. They confirmed also the earlier findings of Popp and his co-workers, showing again important differences between normal and cancer cells $[20,22]$.

From all these investigations it can be concluded, that merely a minuscule portion (nearly $2 \%$ ) of the hereditary, genetic material (DNA) in the cell nucleus was needed for the build up and regulation of cell processes [22]. All the experiments and computations performed unravel the major task of junk DNA. It seems obvious that these genetic structures, which were believed to be functionless until now, are in charge of the supervision of the highly complex machinery inside the cell by biophotonic action of DNA as proposed by Herbert Fröhlich almost fifty years ago $[8,20]$.

In a co-operation with the photo- and cell biologist Lee LaurentApplegate from the CHUV (Lausanne, Switzerland) we have demonstrated, how the light is captured within the cell and then used for the maintenance of biochemical reactions [30]. These photochemical control processes are crucial part in activation of biochemical reactions as they are well established for almost 40 years in bacterial rhodopsin as well as in the human eye, where photons are trapped by vitamin A. The photoisomerization from 11-cis-retinal to all-trans retinal is induced by a photon. The recharging process back to 11-cis retinal induces a cascade of biochemical reactions as shown in the late 1980's by Lubert Stryer [31].

We have proposed in 2001 [32], that in the human cell, rhodopsin is replaced by the genetic material (DNA). Photodimerization is equivalent to photoisomerization of 11-cis retinal to all trans-retinal. The excision repair process will then activate a cascade of biochemical reactions. The American dermatologist Barbara Gilchrest has 
reported in the mid-nineties of the last century, that sunlight-induced photochemical reactions (the well known pyrimidine photodimers in the DNA) [33] will activate the synthesis of melanin. This skin pigment in the melanocytes of the cutis is responsible for the natural sunlightactivated tanning [32]. This cascade model induced by UV light demonstrates, how photons trigger biochemical reactions and may control them $[30,32]$.

\section{Biophotons May Regulate via Ultraweak Light Impulses Aging Processes of Cells}

Investigations of biophotons within the cell will shed a new light on the conventional cognition of the organism as a part well separated from the environment. It can be substituted by the vision of openness and lucency of the organisms existing in a state of permanent exchange with the environment $[20,34]$.

Therefore in addition to electromagnetic fields there are probably existing further largely unknown and immeasurable fields as they were already proposed by the German anthropologist Carl Huter and reviewed by Castrian [35] and Niggli [36]. Furthermore, the same hypothesis was stated later on by Alexander Gurwitsch $(2,20)$ and Rupert Sheldrake as summarized by Mc Taggart [11]. Huter suggested in 1904 in a previsional manner, that life is based on radiation. In his vision he foretold the light control in the living cell. He established the hypothesis that the physical matter is not merely based on the static (magnetic force in the atomic nucleus) as well as the dynamic energy (electrical power in the electron sheath of the atomic model), but in addition bears a spiritual energy [36]. He positioned this so-called "sensitive energy" as an elementary force into matter. According to his believe, this energy drives the evolution process leading to increasing consciousness from minerals, plants and animals to humans. He proposed that this "sensitive energy" would concentrate more and more during the evolutionary process from elementary particles via atoms and molecules up to the vital force of the living cell in the centriole of eucaryotes. Bornens confirmed the importance of the centriole within the regulation processes of the cell in the year of 1979 [37]. Recommending the "sensitive energy" as a third elementary power besides the static and dynamic energy within the atom, a door was opened to the "subtle fields" as also proposed by Alexander Gurwitsch [20], Albert Einstein and Rupert Sheldrake as reviewed by Mc Taggart [11].

Additionally, the life force "Chi" in old Chinese Medicine belongs to this so-called "subtle fields". Similar views are reported in many medical traditions of the human culture all over the history of humans [38]. The occidental medicine, from Hippocrates (ca. 460BC- ca. 370 BC), the ancient Greek physician as father of western medicine, up to Paracelsus (1493-1541) is based on the speculation of the existence of a vital force It was thought to be the principal force of medical practitioners to induce the healing power in humans $[1,36,38]$. The ultraweak radiation in cells seems to be tightly coupled with this vital force in all creatures. Biophotons represent the potential information and are a physical quantity to be measured [2,27-29,38-45]. In short, as a synthesis of biophotonic research known so far, it may be concluded that ultraweak photons in cells are the carrier of all information within the cell and the organisms. We have previously found differences in ultraweak photon emission after UVA laser induction in human skin fibroblasts originated from young and adult donors [41]. Ultraweak photons may serve as a marker of aging and differentiation as we already have found for the biochemical marker ornithine decarboxylase more than twenty years ago $[17,46]$. We have recently shown that biophotonic emission is closely connected to the differentiation processes in cellular systems [41]. From the results shown a few years ago [32,40,41], it may well be that ultraweak photonic changes in aging cells will serve as molecular signals as shown by Voeikov [26] in 2001. They may regulate by coherent states as predicted by Herbert Fröhlich in the early 1950s and reviewed by Hyland recently [43]. In this concept coherent states of water in living organisms may be highly important $[42,44]$. Therefore, biophotonic signals may well serve as the language of cells. Further intense research on ultraweak photon emission is needed to unravel other secrets in human life and their complex processes in aging and vital activities in general.

\section{Acknowledgements}

I would like to thank Professor Lee Ann Laurent-Applegate (CHUV, Lausanne, Switzerland) as well as Dr. Max Bracher (BioFoton AG, Treyvaux, Switzerland) for critical reading of the manuscript and many helpful discussions. This work is dedicated to the $75^{\text {th }}$ birthday of Professor Fritz-Albert Popp on $11^{\text {th }}$ May 2013 , the German pioneer of biophotonic research who introduced the basis of quantum physics into modern biology.

\section{References}

1. Hellemans A, Bunch B (1988) The timetable of Science, Simon \& Schuster, New York.

2. Popp FA, Beloussov, LV (2003) Integrative Biophysics: Biophotons, Kluwer Academic Publishers, Boston.

3. Pagels HR (1982) The cosmic code. Quantum Physics as the language of nature, Simon \& Schuster, New York.

4. Parrish JA, Anderson RR, Urbach F, Pitts D (1978) UV-A biological effects of ultraviolet radiation with emphasis on human response to long wave radiation Plenum Press, New York.

5. Harm W (1980) Biological effects of ultraviolet radiation, Cambridge University Press, New York.

6. Niggli HJ (1983) Formation and Excision of cyclobutane-type pyrimidine dimers in human skin fibroblasts after irradiation with ultraviolet light. Doctoral thesis in Sciences. University of Lausanne, Switzerland.

7. Rodemann HP, Bayreuther K, Pfleiderer G (1989) The differentiation of norma and transformed human fibroblasts in vitro is influenced by electromagnetic fields. Exp Cell Res 182: 610-621.

8. Hyland GJ (2000) Physics and biology of mobile telephony. Lancet 356: 18331836.

9. Einstein A (1920) Äther und Relativitätstheorie, Rede gehalten am 5. Mai 1920 an der Reichs-Universität Leiden. Berlin.

10. Lambrecht $A$ (2002) Observing mechanical dissipatin in the quantum vacuum: an experimental challenge in Laser physics at the limits, Berlin/New York, Springer.

11. Mc Taggert $L$ (2003) The Field: The Quest for the Secret Force of the Universe Harper Collins Publisher, New York.

12. Puthoff HE (1989) Gravity as a zero-point-fluctuation force. Phys Rev A 39: 2333-2342.

13. Hayflick L, Moorhead PS (1961) The serial cultivation of human diploid cell strains. Exp Cell Res 25: 585-621.

14. Cleaver JE (1968) Defective repair replication of DNA in xeroderma pigmentosum. Nature 218: 652-656.

15. Niggli HJ, Bayreuther K, Rodemann HP, Röthlisberger R, Francz PI (1989) Mitomycin C-induced postmitotic fibroblasts retain the capacity to repair pyrimidine photodimers formed after UV-irradiation. Muta Res 219: 231-240.

16. Niggli HJ (2003) Temperature dependence of ultraweak photon emission in fibroblastic differentiation after irradiation with artificial sunlight. Indian J Exp Biol 41: 419-423.

17. Niggli HJ, Francz PI (1992) May ultraviolet light-induced ornithine decarboxylase response in mitotic and postmitotic human skin fibroblasts serve as a marker of aging and differentiation? Age 15: 55-60.

18. Niggli HJ (1993) Aphidicolin inhibits excision repair of UV-induced pyrimidine 
photodimers in low serum cultures of mitotic and mitomycin C-induced postmitotic human skin fibroblasts. Mutat Res 295: 125-133.

19. Nagl W, Popp FA (1983) A physical (electromagnetic) model of differentiation 1. Basic considerations. Cytobios 37: 45-62.

20. Chang JJ, Fisch J, Popp FA (1998) Biophotons, Kluwer Academic Publishers, Boston.

21. Colli L, Facchini U, Guidotti G (1955) Further measurements on the bioluminescence of the seedlings, Experientia, 11: 479-481.

22. Niggli HJ (1992) Ultraweak photons emitted by cells: biophotons. J Photochem Photobiol B 14: 144-146.

23. Ruth B (1977) Experimenteller Nachweis ultraschwacher Photonenemission aus biologischen Systemen. Doctoral Thesis, University of Marburg, Germany.

24. Cohen S, Popp FA (1997) Biophoton emission of the human body. J Photochem Photobiol B 40: 187-189.

25. Popp FA, Nagl W, Li KH, Scholz W, Weingärtner O, et al. (1984) Biophoton emission. New evidence for coherence and DNA as source. Cell Biophys 6 : $33-52$

26. Voeikov V (2001) Reactive oxygen species, water, photons and life. Rivista di Biologia/Biology Forum, 94193.

27. van Wijk E, Kobayashi M, van Wijk R, van der Greef J (2013) Imaging of ultraweak photon emission in a rheumatoid arthritis mouse model. PLoS One 8 : e84579.

28. Niggli HJ, Tudisco S, Privitera G, Applegate LA, Scordino A, et al. (2005) Laserultraviolet-A-induced ultraweak photon emission in mammalian cells. J Biomed Opt 10: 024006

29. Musumeci F, Privitera G, Scordino A, Tudisco S, Lo Presti C, et al. (2005) Discrimination between normal and cancer cells by using analysis of delayed luminescence. Applied Physics Letters 86: 153902 (3 pages).

30. Applegate LA, Scaletta C, Panizzon R, Niggli H, Frenk E (1999) In vivo induction of pyrimidine dimers in human skin by UVA radiation: initiation of cell damage and/or intercellular communication? Int J Mol Med 3: 467-472.

31. Stryer $L$ (1987) The molecules of visual excitation. Sci Am 257: $42-50$

32. Scaletta C, Popp FA, Niggli HJ, Applegate LA, Yan Yu (2001) UV-induced DNA damage and ultraweak photon emission in human fibroblastic skin cells: parameters to trigger intra- and extra-cellular photobiostimulation. Trends in Photochemistry \& Photobiology 8: 53-65
33. Eller MS, Yaar M, Gilchrest BA (1994) DNA damage and melanogenesis Nature 372: 413-414.

34. Niggli HJ (2003) Temperature dependence of ultraweak photon emission in fibroblastic differentiation after irradiation with artificial sunlight. Indian J Exp Biol 41: 419-423.

35. Castrian W (2004) Lehrbuch der Psycho-Physiognomik, Karl F. Haug Verlag, Stuttgart, Germany.

36. Niggli HJ (1998) Biophotons: our body produces light, Network 68: 16-17.

37. Bornens M (1979) The centriole as a gyroscopic oscillator. Implications fo cellorganization and some other consequences. Biologie Cellulaire 35: 115132.

38. Schlebusch KP, Maric-Oehler W, Popp FA (2005) Biophotonics in the infrared spectral range reveal acupuncture meridian structure of the body. $\mathrm{J}$ Altern Complement Med 11: 171-173.

39. Lenger K, Bajpai RP, Spielmann M (2013) Identification of Unknown Homeopathic Remedies by Delayed Luminescence. Cell Biochem Biophys.

40. Niggli HJ, Tudisco S, Privitera G, Applegate LA, Scordino A, (2007) News and Views in UVA-laser induced ultraweak delayed luminescence of cultred cells. Springer New York.

41. Niggli HJ, Tudisco S, Lanzanò L, Applegate LA, Scordino A, et al. (2008) Laser-ultraviolet-A-induced ultraweak photon emission in human skin cells: A biophotonic comparision between keratinocytes and fibroblasts. Indian J Exp Biol 46: 358-363.

42. Rothe GM (2012) Wasser und Photonen, Michaels Verlag, D-86971 Peiting, Germany.

43. Hyland GJ (2009) Fröhlich's physical theory of cancer- Fröhlichs path from theoretical physics to biology, and the cancer problem. Neural Network World 19: $337-354$

44. Del Giudice E, Elia V, Tedeschi A (2009) Role of water in the living organisms. Neural Network World 19, 355-360.

45. Cifra M, Fields JZ, Farhadi A (2011) Electromagnetic cellular interactions. Prog Biophys Mol Biol 105: 223-246.

46. Niggli HJ, Röthlisberger R (1988) Cyclobutane-type pyrimidine photodimer formation and induction of ornithine decarboxylase in human skin fibroblasts after UV irradiation. J Invest Dermatol 91: 579-584. 